\title{
Cluster randomisation or randomised consent as an appropriate methodology for trials in palliative care: a feasibility study [ISRCTN60243484]
}

\author{
Andrew Fowell ${ }^{1}$, Ian Russell2 ${ }^{2}$, Ros Johnstone*1, Ilora Finlay ${ }^{3}$ and \\ Daphne Russell ${ }^{2}$
}

Address: ${ }^{1}$ Palliative Care Department North west Wales NHS Trust Bodfan Eryri Hospital Caernarfon Gwynedd LL55 2YE Wales, UK, ${ }^{2}$ Institute of Medical and Social Care Research Wheldon Building University of Wales Bangor Gwynedd LL57 2UW Wales, UK and ${ }^{3 V i c e}$ Dean University of Wales college of Medicine Heath Park Cardiff CF14 4XN Wales, UK

Email: Andrew Fowell - andrew.fowell@nww-tr.wales.nhs.uk; Ian Russell - ian.russell@bangor.ac.uk; Ros Johnstone* - wccpp.manager@nwwtr.wales.nhs.uk; Ilora Finlay - ilora.finlay@velindre.tr.wales.nhs.uk; Daphne Russell - d.russell@bangor.ac.uk

* Corresponding author

Published: 27 April 2004

BMC Palliative Care 2004, 3:I
Received: 23 March 2004

Accepted: 27 April 2004

This article is available from: http://www.biomedcentral.com/l472-684X/3/I

(c) 2004 Fowell et al; licensee BioMed Central Ltd. This is an Open Access article: verbatim copying and redistribution of this article are permitted in all media for any purpose, provided this notice is preserved along with the article's original URL.

\begin{abstract}
Background: Although guidelines for the care of the dying patient exist the evidence base to support the guidelines is poor. Some of the factors contributing to this include failure to recruit to trials, protective healthcare professionals and subsequent attrition from trials due to the death of the patients. Recent studies report favourably on the use of cluster randomisation as an appropriate methodology for use in this patient group.
\end{abstract}

Methods/design: A feasibility study, exploring two types of randomisation as appropriate methodology for trials involving dying patients. Cluster randomisation and randomised consent will be utilised following a crossover design at two sites, one oncology ward and one Macmillan unit within the Northwest Wales NHS Trust. All patients commencing on the Integrated Care Pathway (ICP) for the Last Days of Life will be eligible for inclusion in the study. Using the hypothesis that it is not necessary to prescribe an anti-emetic medication when setting up a syringe driver for the dying patient, the study will evaluate different models of research methodology.

Discussion: The identification of the most appropriate methodology for use in studies concerning this patient group will inform the development of future clinical studies. Furthermore, the outcomes of this feasibility study will inform the development, of a proposal seeking funding for Wales-wide trials in palliative care. The identification of an appropriate methodology will provide a starting point for the establishment of a robust evidence base for the care of the dying patient.

\section{Background}

Designing trials involving dying patients can be fraught with methodological issues and ethical considerations since this is a vulnerable patient group $[1,2]$. Within palliative care there is a significant lack of research evidence with respect to the treatment and care of the dying patient
[3]. Previous studies [4] have detailed inherent difficulties in carrying out methodologically sound research at the end of life. Difficulties recruiting to trials are documented, [5] and attrition from trials due to death is recognised as problematic [6]. 
Guidelines for the care of the dying patient exist, [7] however, the evidence base to support the guidelines is poor. In England the implementation of the Liverpool integrated care pathway for the last two days of life has facilitated the production of data for research and audit purposes [8]. The Wales-wide implementation of an integrated care pathway (ICP) for the last two days of life has facilitated local and national benchmarking exercises [9]. Analysis of 2500 variance sheets from patients dying on the ICP across Wales indicates that, there is little evidence to support the routine use of anti-emetic medication in syringe drivers for dying patients. Furthermore, the inclusion of an anti-emetic in the syringe driver in combination with other medications can result in crystallisation [10] consequently, the medications may not be safely and efficiently delivered to the patient.

Recent studies seeking to investigate an intervention in palliative care report favourably on the use of a cluster randomised design as an alternative method for establishing evidence of effectiveness in this specific patient group [11]. Utilising the cluster randomisation methodology, consent can be sought at cluster level through the appointment of a cluster gatekeeper [12]. The cluster guardian witnesses the cluster consent given by the gatekeeper and holds a supervisory role for the duration of the study. Both the cluster gatekeeper and the cluster guardian must be independent of the research team. The cluster gatekeeper may after due consideration and consultation with the cluster guardian withdraw the cluster from the study.

Randomised or Zelen consent hereafter referred to as " Zelen" [13] as an alternative methodology seeks informed consent at the onset of treatment and after randomisation if the patient is to receive the experimental treatment. Patients consenting to the trial treatment have the right to withdraw from the trial at any time.

One of the main criticisms of Zelen's method is that a consideration of sample size needs to be undertaken if the study is not to be undermined by patients opting out, or declining to be recruited $[14,15]$. There are specific ethical issues associated with the design and conduct of Zelen trials [16]. Collaboration between palliative care researchers and ethics committees to develop palliative care studies is advocated in the literature [17]. This paper outlines a feasibility study to explore cluster randomisation and randomised consent as viable methodology for future trials involving dying patients. The study will take place over one year in two sites, one oncology ward and one Macmillan unit within the Northwest Wales NHS Trust and employ a crossover design. The outcomes of the study will inform the development of a full proposal to undertake a Wales-wide trial seeking collaboration with the Welsh
Cancer Trials Network and the National Cancer Research Institute.

\section{Method}

Ethical approval for this feasibility study was granted by the North Wales Health Authority Research Committee (West, Central and East sub-committees).

Two sites will participate in the study, an oncology ward in a local district general hospital, and the Macmillan unit in a local community hospital. The two sites will be randomly allocated to begin the study with either the Zelen method or the cluster randomisation method, allocation will be undertaken independently and from a distance by the University of Wales Bangor. On admission to either ward on each site patients will be approached for consent to use their medical data (See additional file 1).

\section{Zelen method}

The procurement of informed consent from the dying patient to participate in a palliative care trial will be approached pragmatically. The design of the trial necessitates that informed consent is required in those patients randomised to the experimental arm of the study only. At the commencement of the syringe driver, the patient in the experimental arm of the study will be informed of the procedure to omit the anti-emetic from the syringe driver (See additional file 2), and invited to give written consent to participate in the study (See additional file 3 ). The patient will be reassured that if they vomit or feel at all nauseous an anti-emetic will be available and administered. The patient will also be made aware that if at any time they wish to withdraw from the study they are free to do so with no repercussions for their care or comfort.

Participation in the trial entails minimal risk to the patient and endeavours to reduce the pharmacological burden on the dying patient. Furthermore, the omission of an antiemetic in the syringe driver may result in enhanced safety and efficiency of medication delivery.

\section{Cluster randomisation}

Consent for inclusion in the cluster randomisation trial will be sought at cluster level rather than on an individual basis. As a cluster representation mechanism (CRM) a cluster gatekeeper will be designated. The cluster gatekeeper will be informed by letter of the study aims and methods and invited to act as cluster gatekeeper (See additional file 4). As cluster gatekeeper the designated healthcare professional will be responsible for 'assenting' his/her cluster to the trial (See additional file 5). The cluster gatekeeper provides informed assent for the cluster to participate in the study or trial. The cluster guardian is informed of the study and is recruited from management within the North West Wales NHS Trust. After due consideration and 
consultation with the cluster guardian, the gatekeeper may withdraw the cluster from the trial. Any instances of withdrawal will be fully documented and reported.

There are two sites or clusters in this study therefore, two cluster gatekeepers will be appointed. The cluster gatekeepers can be appointed from key nursing or medical staff at the two sites, but must be independent of the research team. The cluster gatekeepers are responsible to the cluster in the first instance and answerable to the cluster guardians.

The cluster guardians will be drawn from management within the Northwest Wales NHS Trust. Management staff at both sites will be contacted by letter and informed of the study, the same letter will carry an invitation to act as cluster guardian for the duration of the study (See additional file 6). The cluster guardians will witness the cluster assent given by the cluster gatekeepers. The cluster guardians must be independent of the research team.

This study is an exploration of cluster randomisation and Zelen as an appropriate methodology for studies involving dying patients and does not on this occasion seek to reach statistical significance with any outcomes of the study. To the same end, the sample sizes and subsequent power of the design are not of primary concern, the main issue of interest in this study is the appropriateness of the methodology for further studies in palliative care. However, it is expected that the study will highlight any inherent difficulties in recruiting to trial and identify the potential flaws in achieving optimum sample size for the study design.

It will be important to inform palliative care staff at the two participating sites as to the nature of the trial (See additional file 7). Prior to the onset of the active phase of the study, a baseline assessment of the use of anti-emetic medications in syringe drivers, for patients on the integrated care pathway for the last days of life will be undertaken.

The project nurse will give a general introduction to the study at both sites and after each site has been randomised to one of the two methods, the project nurse will then revisit each site and give a more specific presentation regarding the methodology. Handouts of the presentations will be left with staff at both sites and three months later repeated when the two sites swap methodologies. To maintain consistency in the way that the two sites are introduced to the study and the different methods, a "script" will be developed and used as a framework for the presentations.
Two hospital sites with palliative care beds within the Northwest Wales NHS Trust will be randomly allocated one of two methodological conditions, cluster randomisation or Zelen. Both sites will have two treatment conditions, to routinely prescribe an anti-emetic at the commencement of a syringe driver for a dying patient, the usual procedure, or to change practice and not prescribe an anti-emetic when commencing on a syringe driver. However, if the patient experiences nausea or vomiting then medication will be available and administered immediately. With respect to the Zelen method, the patient is randomised to the experimental treatment (Condition A) through the use of a "Decision Tree" (See additional file 8).

Each site will continue with the designated methodology for three months, the project nurse will regularly visit each site and request the medical notes of each patient dying on the care pathway. At the end of a three-month period, the project nurse will hold a 'de-briefing' session at each site, prior to introducing the new method for the next three months. After three months the methodology at the two sites will be switched or crossed over so that site one, previously employing cluster randomisation as a methodology will now be utilising Zelen and vice versa (See additional file 9). Each site will be monitored to assess progress, and to identify any difficulties in adhering to the study protocol. Patients who have been prescribed antiemetics in the immediate period prior to the commencement of a syringe driver, will form a sub-set of the study. Both sites will record any variances to the agreed course of care on the pathway variance sheet.

\section{Outcomes}

After a trial of each method for three months at each site, the appropriateness of cluster randomisation and Zelen as a methodology for trials in palliative care will be examined and the facilitators and inhibitors identified. The rate of refusal to recruit to the new treatment in the Zelen model is an issue raised in the literature $[14,15]$.

Considerations will be given to issues of sample size and statistical power to inform the development of prospective studies. Further evaluation of the variance report as a tool for obtaining detailed information from the ICP will be undertaken.

The variance reports will be analysed and compared, to establish if there is any clear evidence to support the cessation of routine prescribing of anti-emetic medication in syringe drivers for dying patients.

Combinations of analgesics and anti-emetics can precipitate in syringe drivers causing administrative errors and inadequate periods of analgesia. Exploring the necessity 
of routine prescribing of anti-emetics in syringe drivers could reduce the pharmacological burden on the dying patient and improve the safety and efficiency with which medications are delivered to the patient.

Explorations of Zelen and cluster randomisation, as appropriate methods for future studies involving dying patients are important. At present the evidence base for the care of this specific patient group is poor. The identification of the most appropriate methodology for use in studies concerning this patient group will inform the development of future clinical studies.

An identification of the factors contributing to attrition rates in a study concerning dying patients will be valuable. The outcomes of this feasibility study will inform future studies about the sample size needed to reach statistical power for this particular research design and the specific patient population.

The utility of the variance sheet as a data-reporting tool within a trial environment will be explored.

Furthermore, the outcomes of this feasibility study will inform the development, of a proposal seeking funding for Wales-wide trials in palliative care. The identification of an appropriate methodology will provide a starting point for the establishment of a robust evidence base for the care of the dying patient.

\section{Competing interests}

None declared.

\section{Authors' contributions}

AF, RJ \&IR participated in the design of the study and editing the manuscript.

DR was responsible for the randomisation of the two sites and the randomisation of patients with respect to the 'Zelen' method and editing the manuscript.

IF participated in editing the manuscript

\section{Additional material}

\section{Additional File 1}

Patient consent to use data

Click here for file

[http://www.biomedcentral.com/content/supplementary/1472684X-3-1-S1.doc]

\section{Additional File 2}

Zelen method patient information

Click here for file

[http://www.biomedcentral.com/content/supplementary/1472-

684X-3-1-S2.doc]

\section{Additional File 3}

Zelen consent

Click here for file

[http://www.biomedcentral.com/content/supplementary/1472684X-3-1-S3.doc]

\section{Additional File 4}

Cluster gatekeeper invitation

Click here for file

[http://www.biomedcentral.com/content/supplementary/1472684X-3-1-S4.doc]

\section{Additional File 5}

\section{Cluster gatekeeper assent}

Click here for file

[http://www.biomedcentral.com/content/supplementary/1472684X-3-1-S5.doc]

\section{Additional File 6}

\section{Cluster guardian invitation}

Click here for file

[http://www.biomedcentral.com/content/supplementary/1472684X-3-1-S6.doc]

\section{Additional File 7}

Healthcare staff information sheet

Click here for file

[http://www.biomedcentral.com/content/supplementary/1472684X-3-1-S7.doc]

\section{Additional File 8}

Randomisation decision tree

Click here for file

[http://www.biomedcentral.com/content/supplementary/1472684X-3-1-S8.doc]

\section{Additional File 9}

Timetable of study

Click here for file

[http://www.biomedcentral.com/content/supplementary/1472684X-3-1-S9.doc]

\section{References}

I. Ellershaw J, Ward C: Care of the Dying Patient: the last hours or days of life. British Medical Journal 2003, 326:30-34.

2. Hardy J: Consent for Trials in Palliative Care. The Lancet 2000, 356 Suppl:S44.

3. Higginson I: Evidence Based Palliative Care. British Medical Journal 1999, 31 9:462-63.

4. Jordhoy MS, Fayers P, Saltnes T, Ahler-Elmqvist M, Kaasa S: Palliative Care Intervention and Death at Home: a Cluster Randomised Trial. The Lancet 2000, 356:888-893. September 92000

5. Cook AM, Finlay IG, Butler-Keating RJ: Recruiting into Palliative Care Trials: Lessons Learnt from a Feasibility Study. Palliative Medicine 2002, 16:163-165.

6. Grande GE, Todd Cl: Why are Trials in Palliative Care so Difficult. Palliative Medicine 2002, 14:69-74.

7. "Changing Gear". Working party on Clinical Guidelines in Palliative Care National Council for Hospice and Specialist Palliative Care Services London 1997.

8. Ellershaw J, Ward C: Care of the Dying Patient: the last hours or days of life. British Medical Journal 2003, 326:30-34. 
9. Fowell A, Finlay IG, Johnstone R, Minto L: An Integrated Care Pathway for the Last Two Days of Life: Wales-wide Benchmarking in Palliative Care. International Journal of Palliative Nursing 2002, 8( I 2):566-573.

10. Back IN: Palliative Medicine Handbook. Cambrian Aberystwyth Ceredigion 3200I.

II. Jordhoy MS, Fayers P, Saltnes T, Ahler-Elmqvist M, Kaasa S: A Palliative Care Intervention and Death at Home: a Cluster Randomised Trial. The Lancet 2000, 356:888-893.

12. Medical Research Council: Cluster Randomised Trials: Methodological and Ethical Considerations. MRC Clinical Trials Series MRC London UK 2003 [http://www.mrc.ac.uk].

13. Zelen M: Randomised Consent Designs for Clinical Trials: an Update. Statistical Medicine 1990, 9:645-656.

14. Torgerson DJ: Commentary: Problems with Randomised Consent. British Medical Journal 200I, 322:453-460.

15. Steiner A, Walsh B, Pickering R, Wiles R, Ward J, Brooking J: Therapeutic Nursing or Unblocking Beds? A Randomised Controlled Trial of a Post Acute Intermediate Care Unit. British Medical Journal 200I, 322:453-460.

16. Torgerson DJ: Contamination in Trials: is Cluster Randomisation the Answer? British Medical Journal 200I, 322:355-357.

17. Edwards SJ, Braunholtz DA, Lilford RJ, Stevens AJ: Ethical issues in the design and conduct of cluster randomised controlled trials. British Medical Journal 1999, 31 8: I 407-I409.

18. Cassarette K, Karlawish J: Are Special Guidelines Needed for Palliative Care Research? Journal of Pain and Symptom Management 2000, 20(2):130-139.

\section{Pre-publication history}

The pre-publication history for this paper can be accessed here:

http://www.biomedcentral.com/1472-684X/3/1/prepub

\section{Publish with Bio Med Central and every scientist can read your work free of charge}

"BioMed Central will be the most significant development for disseminating the results of biomedical research in our lifetime. "

Sir Paul Nurse, Cancer Research UK

Your research papers will be:

- available free of charge to the entire biomedical community

- peer reviewed and published immediately upon acceptance

- cited in PubMed and archived on PubMed Central

- yours - you keep the copyright

Submit your manuscript here:

http://www.biomedcentral.com/info/publishing_adv.asp 